\title{
A New Evolutionary Decision Theory for Many-Objective Optimization Problems
}

\author{
Zhuo Kang ${ }^{1}$, Lishan Kang ${ }^{2,3} *$, Xiufen Zou ${ }^{4}$, Minzhong Liu ${ }^{5}$, Changhe $\mathrm{Li}^{2}$, \\ Ming Yang ${ }^{2}$, Yan $\mathrm{Li}^{1}$, Yuping $\mathrm{Chen}^{3}$, and Sanyou Zeng ${ }^{2}$ \\ ${ }^{1}$ Computation Center, Wuhan University, Wuhan 430072, China1 \\ kang_whu@yahoo.com \\ ${ }^{2}$ School of Computer Science, China University of Geosciences(Wuhan) Wuhan, \\ 430074, China \\ ${ }^{3}$ State Key Laboratory of Software Engineering, Wuhan University, Wuhan 430072, China \\ ${ }^{4}$ School of Mathematics and Statistics, Wuhan University, Wuhan 430072, China \\ ${ }^{5}$ School of Computer Science, Wuhan University, Wuhan 430072, China
}

\begin{abstract}
In this paper the authors point out that the Pareto Optimality is unfair, unreasonable and imperfect for Many-objective Optimization Problems (MOPs) underlying the hypothesis that all objectives have equal importance. The key contribution of this paper is the discovery of the new definition of optimality called $\varepsilon$-optimality for MOP that is based on a new conception, so called $\varepsilon$-dominance, which not only considers the difference of the number of superior and inferior objectives between two feasible solutions, but also considers the values of improved objective functions underlying the hypothesis that all objectives in the problem have equal importance. Two new evolutionary algorithms are given, where $\varepsilon$ - dominance is used as a selection strategy with the winning score as an elite strategy for search -optimal solutions. Two benchmark problems are designed for testing the new concepts of many-objective optimization problems. Numerical experiments show that the new definition of optimality is more perfect than that of the Pareto Optimality which is widely used in the evolutionary computation community for solving many-objective optimization problems.
\end{abstract}

Keywords: Many-objective optimization; Pareto optimality; $\varepsilon$-optimality; $\varepsilon$-dominance.

\section{Introduction}

Most optimization problems in nature have many objectives (normally conflicting with each other) that need to be optimized at the same time. These problems are called Multi-Objective Problems (MOPs), which are studied in economies, sciences and engineering. People realize the importance of solving MOPs and the development of

* Corresponding author. 
theory and methodology to deal with this kind of problems become an important area of computational intelligence. Because of the conflicting nature of their objectives, MOP does not normally have a single solution and, in fact, they even require the definition of a new notion of "optimality." The most commonly adopted notion of optimality in MOPs is that originally proposed by Edgeworth ${ }^{[1]}$ and later generalized by Pareto ${ }^{[2]}$. Such a notion is called Edgeworth-Pareto Optimality or, more commonly, Pareto Optimality $^{[3]}$.

There are two ways for solving MOPs to find their Pareto optimal sets. One is using the weighted objectives summed in one objective. Another is using the population search strategies. The former is called "One Many" and the later is called "Many Once"[4]. Why evolutionary multi-objective optimization algorithms are increasingly inappropriate as the number of objectives increases? Can we find other ways for solving many-objective optimization problems? Recently, Maneeratana, Boonlong, and Chaiyaratana ${ }^{[5]}$ proposed a method called Compressed-Objective Genetic Algorithm (COGA) for solving an optimization problem with a large number of objectives by transforming the original objective vector into a two-objective vector during survival selection is presented. The transformed objectives, referred to as preference objectives, consist of a winning score and a vicinity index. The winning score, a maximization criterion, describes the difference of the number of superior and inferior objectives between two solutions. The vicinity index, a minimization criterion, describes the level of solution clustering around a search location which is used to encourage the results to spread throughout the Pareto front. The new conception in that paper is the definition of Winning Score, a preference objective which concerns numbers of superior and inferior objectives between a pair of two non-dominated solutions. But the goal of the authors is still to get a solution set which is coverage and closeness to the true Pareto front.

In 2003, Zhou, Kang, Chen and Huang ${ }^{[6]}$ proposed a new definition (Evolving Solutions) for MOP to answer the essential question: what is a multi-objective optimal solution and advance an asynchronous evolutionary model, Multiple Island Net Training Model (MINT model), to solve MOPs, especially to solve many-objective optimization problems. The new theory is based on their understanding of the natural evolution and the analysis of the difference between natural evolution and MOP, thus it is not only different from the tradition methods of weighted objective sum, but also different from Pareto Optimization.

Benedelti, Farina, and Gobbi ${ }^{[7]}$ mentioned three reasons of Pareto definition of optimality which can be unsatisfactory to a large number of objective optimization.

$\mathrm{P} 1$, The number of improved objective functions values is not taken into account.

$\mathrm{P} 2$, The (normalized) relevance of the improvements is not taken into account.

P3, No preference among objectives is considered.

They give two new definitions to meet above issues. One is called k Optimality when taking into account number of improved objectives. Another is called Fuzzy Optimality when taking into account size of improvements and including parameters to be provided by decision maker with the underlying hypothesis that all objectives have equal importance. 
We must point out that the definition of Pareto Optimality is fair, reasonable and perfectly correct for two-objective optimization problems, but is unfair, unreasonable and imperfect for more than two objectives which are in strict conflict of each other, especially for a large number of objectives, if all objectives have equal importance.

Kang ${ }^{[8]}$ gives an example:

Minimize $F(x) \underline{\underline{\Delta}}\left(f_{1}(x), f_{2}(x), \cdots, f_{m}(x)\right)$

where $\mathrm{m}$ is a large number,

and $f_{i}(x)=(x-i)^{2}, i=1,2, \ldots, m, \quad 1 \leq x \leq m$.

It is clear that its Pareto Optimal Set is $[1, \mathrm{~m}]$ which is the exact decision space of the MOP. It is not clear at all what a decision maker can do with such a large result of a Pareto Optimal Set in practice!

In this paper, a new definition of optimality for MOPs is given. It not only considers the number of improved objectives, but also considers the convergence of improved values of objective functions. The remainder of this paper is organized as follows. The new definitions of optimality are presented in section 2. The new evolutionary algorithms are given in Section 3. Section 4 presents experimental design, benchmarks and results for the new definitions of optimality. Section 5 is conclusions and new research directions for future MOPs research with the new concepts proposed by this paper.

\section{What Is the Optimality for MOP?}

A MOP can be described as follows.

Let $D \subseteq R^{n}$ be decision variable space and $O \subseteq R^{m}$ be objective space.

Let F: $D \rightarrow O, \mathrm{~g}: D \rightarrow R^{p}$ and $h: D \rightarrow R^{q}$ be vector functions.

A nonlinear constrained multi-objective optimization problem (MOP) is defined as

$$
\min _{x \in S} f(x) \underline{\underline{\Delta}}\left(f_{1}(x), f_{2}(x), \cdots, f_{m}(x)\right)
$$

where

$$
\begin{aligned}
S & =\{X \in D \mid g(X) \leq 0 \wedge h(X)=0\} \\
D & =\{L \leq X \leq U\},
\end{aligned}
$$

where $\quad L, U \in R^{n}$ are given.

We first normalize the MOP (1) as follows.

Denote

$$
\min _{x \in S} f_{i}(x)=f_{i}^{\min } \text { and } \max _{\mathrm{x} \in \mathrm{S}} f_{i}(x)=f_{i}^{\max } \forall i \in M=\{1,2, \cdots, m\}
$$

Let $F_{i}(x)=\left(f_{i}(x)-f_{i}^{\min }\right) /\left(f_{i}^{\max }-f_{i}^{\min }\right) \quad \forall i \in M \quad$ be transformations. 
The MOP (1) is transformed into

$$
\min _{x \in S} F(x) \triangleq\left(F_{1}(x), F_{2}(x), \cdots, F_{m}(x)\right)
$$

where $\mathrm{F}: D \rightarrow O=[0,1]^{m}$, the objective space.

For each pair of variables $X_{1}, X_{2} \in S$, three integer functions are defined as follows:

$$
\begin{aligned}
& B_{t}\left(X_{1}, X_{2}\right)=\left|\left\{i \in M \quad F_{i}\left(X_{1}\right)<F_{i}\left(X_{2}\right)\right\}\right| \\
& W_{s}\left(X_{1}, X_{2}\right)=\left|\left\{i \in M \quad F_{i}\left(X_{1}\right)>F_{i}\left(X_{2}\right)\right\}\right| \\
& E_{q}\left(X_{1}, X_{2}\right)=\left|\left\{i \in M \quad F_{i}\left(X_{1}\right)=F_{i}\left(X_{2}\right)\right\}\right|
\end{aligned}
$$

where $B_{t}, W_{s}$ and $E_{q}$ mean Better Than, Worse Than and Equivalent To, respectively.

It is clear that $B_{t}+W_{s}+E_{q}=m$ (the number of objectives).

The ideal objective vector of the MOP (2) in objective space is the origin which corresponds to a non-existent solution in general.

The distance from any point $F(X)$ in objective space $\mathrm{O}$ to the ideal objective vector (the origin) is defined as

$$
\|F(X)\|=\sqrt{\sum_{i=1}^{m}\left(F_{i}(X)\right)^{2}}
$$

For any pair $X_{1}, X_{2} \in S$,we give following definitions:

Definition 1 ( $\varepsilon$-dominance): If $\left(B_{t}-W_{s}=\varepsilon>0\right) \wedge\left(\left\|F\left(X_{1}\right)\right\|<\left\|F\left(X_{2}\right)\right\|\right)$ then $X_{1}$ is said to $\boldsymbol{\varepsilon}$-dominate $X_{2}$ (denoted as $\left.X_{1} \prec_{\varepsilon} X_{2}\right)$.

If $W_{s}\left(X_{1}, X_{2}\right)=0$, then $\boldsymbol{\varepsilon}$-dominance is equivalent to Pareto dominance.

Definition 2 ( $\varepsilon$-Optimality): $X^{*} \in S$ is a $\varepsilon$-optimal solution if there is no $X \in S$ such that $\mathrm{X} \boldsymbol{\varepsilon}$-dominates $X^{*}$. (Note: the concept of $\boldsymbol{\varepsilon}$-Optimality is different to the $\varepsilon$ optimal solutions in [13].)

Definition 3 ( $\varepsilon$-Optimal Set and Front): The set of all $\boldsymbol{\varepsilon}$-optimal solutions is called $\boldsymbol{\varepsilon}$-optimal set denoted as $S_{\boldsymbol{\varepsilon}}$ and its range of mapping $F\left(S_{\boldsymbol{\varepsilon}}\right)$ is called $\boldsymbol{\varepsilon}$-optimal front denoted as $F_{\boldsymbol{\varepsilon}}$.

In definition $1, \boldsymbol{\varepsilon}>0$ means that the number of superior objectives is great than the number of inferior objectives between two feasible solutions: $X_{1}$ and $X_{2}$. Because the importance of all objectives are equal, so the definition of $\varepsilon$-dominance is fair and reasonable, and the distance condition: $\left\|F\left(X_{1}\right)\right\|<\left\|F\left(X_{2}\right)\right\|$ not only substantiates the meaning of dominance, but also can be used to control the values of inferior objectives. We must point out that the new definitions of optimality for MOPs are 
imperfect, too, because the transitive property is not existent so the pair $\left([0,1]^{m}, \preceq_{\varepsilon}\right)$ can not form a partially ordered set. For overcoming this difficulty, we use the winning score $^{[5]}$ to rank the individuals of population and archives.

Definition 4 (Winning Score): Let the size of a set A be L, the winning score $W S_{i}$ of the $i^{\text {th }}$ element $\mathrm{X}_{\mathrm{i}}$ in $\mathrm{A}$ is given by

$$
W S_{i}=\sum_{j=1}^{L}\left[B_{t}\left(X_{i}, X_{j}\right)-W_{s}\left(X_{i}, X_{j}\right)\right], \quad i=1,2, \cdots, L
$$

For $X_{i}, i=1,2, \ldots, \mathrm{L}$, we rank them by their winning score from the biggest one to the smallest as $0,1,2, \ldots, k, k \leqslant \mathrm{~L}-1$. The element $\mathrm{X}$ with biggest $W S$ has $\mathrm{RANK}=0$. If there are many elements in A having the same winning score, then they have the same rank.

Definition 5 (minimal element): Element $x \in \mathrm{A}$ is called a minimal element of $\mathrm{A}$, if its rank is 0 .

All minimal elements of A form a minimal element set $\mathrm{M}(\mathrm{A}, \mathrm{RANK}=0)$.

\section{New Evolutionary Algorithms for Solving MOPs}

New algorithms for solving many-objective optimization problems are given as follows.

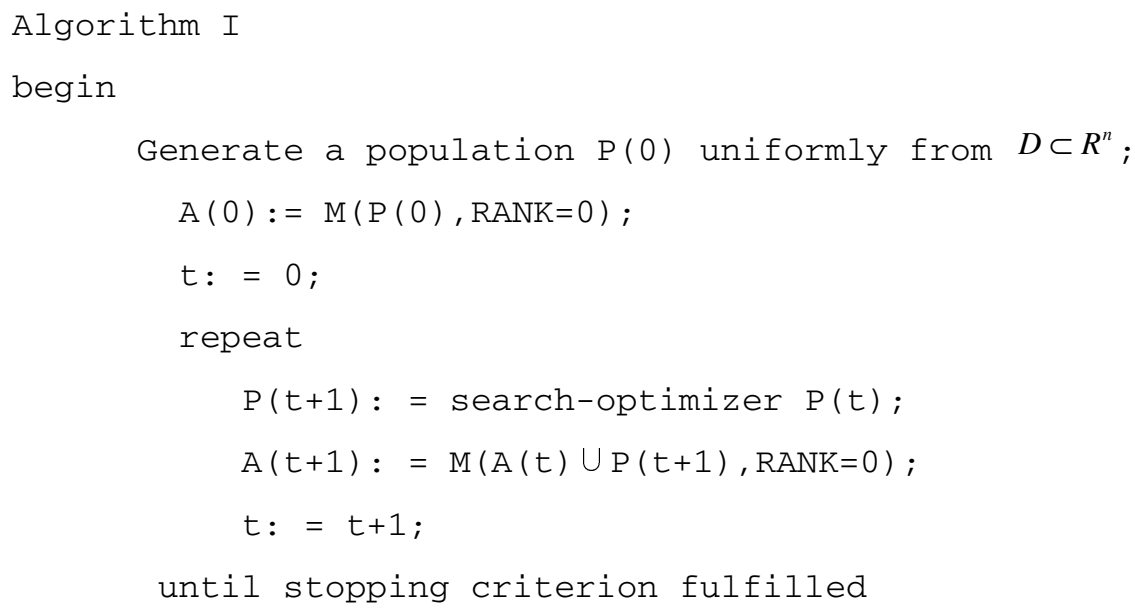

where search-optimizer can be any efficient optimizer which is used for producing offspring. We use the Fast Particle Swarm Optimization Algorithm ${ }^{[12]}$ as search-optimizer which is based on Particle Swarm Optimization with Cauchy mutation and an $\varepsilon$ - optimality based selection strategy. 
Fast Particle Swarm Optimizer

Begin

For $i=1$ to $\mathrm{N}$ do

$\mathrm{Vi}:=\mathrm{Vi}+\eta 1$ rand ()$\quad(\mathrm{Xi}$ best $-\mathrm{Xi})+\eta 2 \operatorname{rand}() \quad(\mathrm{Xg}$

best-Xi);

$$
\begin{aligned}
& X_{i}^{\prime}:=X_{i}+V_{i} ; \\
& V_{i}^{\prime}:=V_{i}^{\prime} \exp \left(\tau_{1} \delta_{1}+\tau_{2} \delta_{2}\right) \\
& X_{i}:=X_{i}+V_{i}^{\prime} \delta_{3} ; \\
& \text { if }\left(\operatorname{better}\left(X_{i}^{\prime}, X_{i}\right) \wedge\left(X_{i} \prec_{\varepsilon} X^{\prime}\right)\right) \text { then } X_{i}:=X_{i}^{\prime} ;
\end{aligned}
$$

endfor

end

where $\delta_{1}, \delta_{2}$ and $\delta_{3}$ are Cauchy random numbers, and $\eta_{1}, \eta_{2}, \tau_{1}$ and $\tau_{2}$ are parameters,

$$
\text { better }(X, Y)= \begin{cases}\text { true } & \|h(X)\|+\bar{g}(X)<\|h(Y)\|+\bar{g}(Y) \\ \text { false } & \|h(X)\|+\bar{g}(X)>\|h(Y)\|+\bar{g}(Y) \\ \text { true } & (\|h(X)\|+\bar{g}(X)=\|h(Y)\|+\bar{g}(Y)) \wedge(\|F(X)\|<\|F(Y)\|) \\ \text { false } & (\|h(X)\|+\bar{g}(X)=\|h(Y)\|+\bar{g}(Y)) \wedge(\|F(X)\| \geq\|F(Y)\|)\end{cases}
$$

where

Algorithm I is almost the same as the Base Algorithm VV in [8].

Algorithm II

begin

Generate a population $\mathrm{P}(0)$ uniformly from $D \subset R^{n}$;

$$
\begin{aligned}
& A(0):=M(P(0), \operatorname{RANK}=0) ; \\
& t:=0 ; \\
& \text { repeat } \\
& \quad P(t+1):=\text { search-optimizer } P(t) ; \\
& \quad A(t+1):=M(A(t) \cup P(t+1), \operatorname{RANK}=0) ; \\
& A(t+1):=\operatorname{Sieve}(A(t+1) ; \\
& \quad t:=t+1 ;
\end{aligned}
$$


until stopping criterion fulfilled

end

where the Fast Particle Swarm Optimization Algorithm $\left.{ }^{[12}\right]$ is used as search-optimizer, and a Sieve procedure is used for control the diversity.

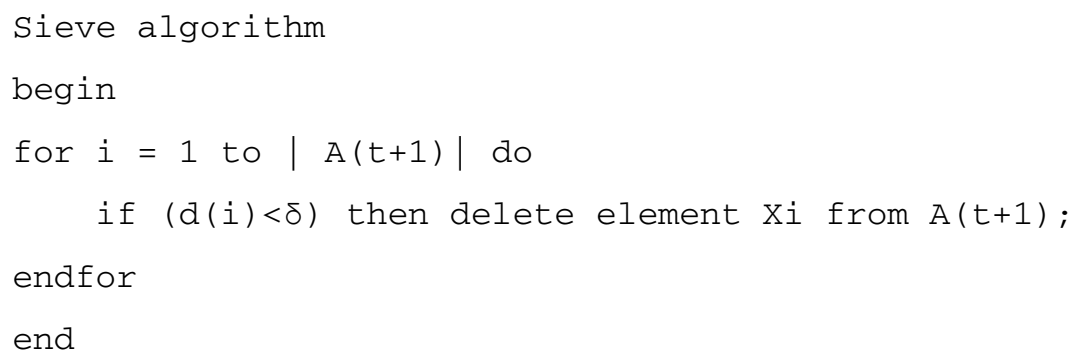

where $d(i)$ is the distance between two nearest points in $A(t+1)$. For more detail, please refer to [11].

Using Algorithm II, a finite well-distributed subset of $M\left([0,1]^{m}, R A N K=0\right)$ can be obtained, because the number of nodes on an $\delta$-net in $[0,1]^{m}$ is finite.

\section{Numerical Experiments}

In order to test the correctness of the new definitions and the effectiveness of Algorithm II, we give two benchmarks ${ }^{[8]}$ with more than three objectives and some numerical results to compare with the results obtained by using Pareto-Optimality.

\subsection{Benchmark Problems}

\section{Benchmark Problem 1}

Minimize $\mathbf{F}(\mathbf{X})=\left(\mathbf{F}_{\mathbf{1}}(\mathbf{X}), \mathbf{F}_{\mathbf{2}}(\mathbf{X}), \ldots, \mathbf{F}_{\mathbf{m}}(\mathbf{X})\right)$ where $F_{i}(X)=(X-i)^{2}, i=1,2, \ldots, m, X \in[1, m]$.

The theoretical Pareto optimal set $S_{p}$ is the whole feasible domain $S=[1, m]$, so it gives us too much information for decision making. However, if we consider $\varepsilon$-optimal set $S_{\varepsilon}$, then we can easily get the following theoretical results:

(1) If $\mathrm{m}$ is even, then its $\varepsilon$-optimal set $S_{\varepsilon}=[\mathrm{m} / 2,(\mathrm{~m}+1) / 2]$.

(2) If $\mathrm{m}$ is odd, then its $\varepsilon$ - optimal set $S_{\varepsilon}=\{(\mathrm{m}+1) / 2\}$, a unique optimal solution.

We will illustrate these results with numerical experiments.

\section{Benchmark Problem 2}

Minimize $\mathrm{F}(\mathrm{X})=\left(f_{1}(x), f_{2}(x), \cdots, f_{9}(x)\right), \quad-2 \leq \mathrm{x} \leq 2, \quad-2 \leq \mathrm{y} \leq 2$, 
where

$$
\begin{aligned}
& f_{1}(x)=-\left(1 /\left(1+\left(\left(\left(x^{2}-y^{2}\right)\left(\left(x^{2}-y^{2}\right)-12 x^{2} y^{2}\right)-1\right)^{2}+4 x^{2} y^{2}\left(3\left(x^{2}-y^{2}\right)^{2}-4 x^{2} y^{2}\right)^{2}\right)^{1 / 2}\right.\right. \\
& +t \sin (x)), \text { where parameter } t \in[-1,1], \\
& f_{2}(x)=\sqrt{(x+1.01)^{2}+y^{2}} \\
& f_{3}(x)=\sqrt{x^{2}+(y+1.01)^{2}} \\
& f_{4}(x)=\sqrt{x^{2}+(y-1.01)^{2}} \\
& f_{5}(x)=\sqrt{(x+0.4825)^{2}+(y+0.8283)^{2}} \\
& f_{6}(x)=\sqrt{(x+0.4825)^{2}+(y-0.8283)^{2}} \\
& f_{7}(x)=\sqrt{(x-0.4825)^{2}+(y-0.8283)^{2}} \\
& f_{8}(x)=\sqrt{(x-0.4825)^{2}+(y+0.8283)^{2}}
\end{aligned}
$$

The first objective $f_{1}(x)$ with parameter $t=0$ is a multi-modal function, which has eight valleys respectively at points : $(0,-1),(0,1),(-1,0),(1,0),(0.47245126882998$, $-0.81830960171391),(0.47245126882998,0.81830960171391),(-0.47245126882998$, $-0.81830960171391),(-0.47245126882998, \quad 0.81830960171391)$

\subsection{Numerical Results}

Benchmark Problem 1. When $m=4$ and $m=5$, the numerical results are depicted on Fig. 1 and Fig.2 respectively. From the figures, we can clearly see that Pareto-optimal set $S_{p}$ is uniformly distributed in the whole feasible domain $S=[1, \mathrm{~m}] ; \varepsilon$-optimal set $S_{\varepsilon}$ is consistent with the above theoretical results .

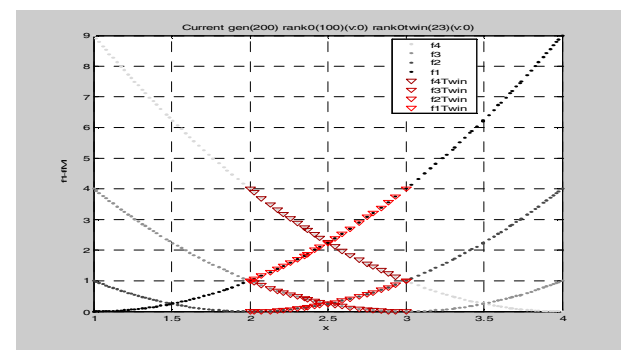

Fig. 1. Comparison of Pareto-front and $\varepsilon$-front for Benchmark Problem 1 with four objectives. (black-dot denote the Pareto-front, red- triangle denote the $\varepsilon$-front.) 


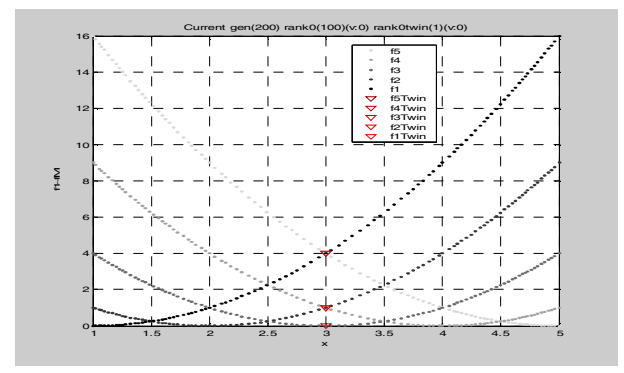

Fig. 2. Comparison of Pareto-front and $\varepsilon$-front for Benchmark Problem 1 with five objectives

\section{Benchmark Problem 2}

From the numerical results(Fig.1, Fig.2, Fig.3(b) and Fig.4(b)), it is found that the number of $\varepsilon$-optimal solutions depends on the number of objectives been odd or even. But compared with the number of Pareto optimal solutions, no matter the number of objectives is odd or even, the number of $\varepsilon$-optimal solutions is quite smaller than that of Pareto optimal solutions.

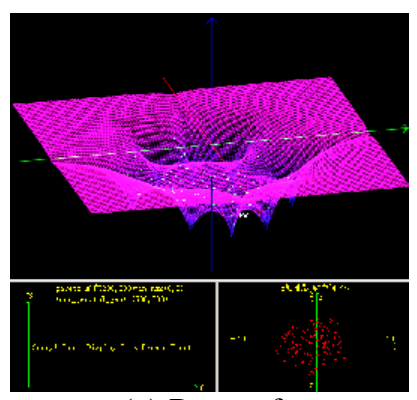

(a) Pareto-front

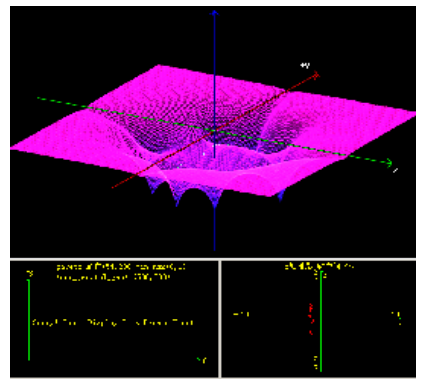

(b) -front

Fig. 3. Comparison of Pareto-front (a) and $\varepsilon$-front (b) with 8 conflict objectives

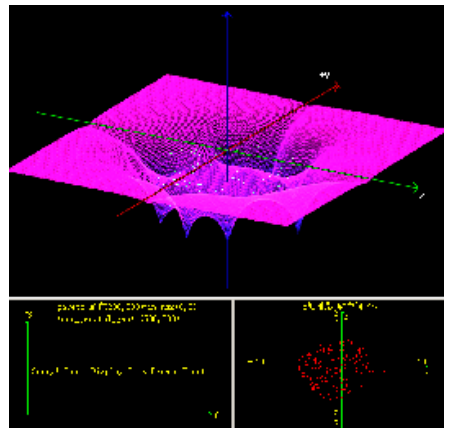

(a) Pareto-front

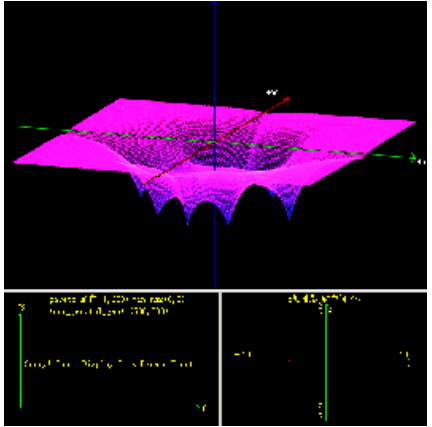

(b) $\varepsilon$-front

Fig. 4. Comparison of Pareto-front (a) and $\varepsilon$-front (b) with 7 conflict objectives 


\section{Conclusions and New Research Directions}

The key contribution in this paper is the new conception of optimality, called $\varepsilon$-optimality, for many-objective optimization. It can be used instead of Pareto-optimality in evolutionary multi-objective optimization algorithms. In the new concept of optimality, the number of objectives is taken into account. It is found that for $\varepsilon=1$ if the number of objectives is odd then the number of $\varepsilon$-optimal solutions is fewer than that with even objectives.

As we mentioned before that the definition of -optimality is imperfect. There exist many difficulties waiting to overcome. For example, how to prove the convergence of the new algorithms? It is believed that the new concept will have a profound and lasting influence in the field of optimization as the applications of the -optimality based evolutionary algorithms being used widely.

Acknowledgements. This work was supported by the National Natural Science Foundation of China (No.60473081) and the Natural Science Foundation of Hubei Province(No. 2005ABA234). Thanks especially give to the anonymous reviewers for their valuable comments.

\section{References}

1. Edgeworth, F.Y.: Mathematical Psychics. P. Keagan, London, UK (1881)

2. Pareto, V.: Cours D'Economie Politique. F. Rouge, Lausanne, Switzerland (1896)

3. Coello Coello, C.A.: Guest Editorial: Special issue on evolutionary multi objective optimization. IEEE Transactions on Evolutionary Computation 7(2), 97-99 (2003)

4. Hughes, E.J.: Evolutionary many-objective optimization: Many once or one many? In: Proceedings of 2005 Congress on Evolutionary Computation, pp. 460-465. IEEE-Press, NJ, New York (2005)

5. Maneeratana, K., Boonlong, K., Chaiyaratana, N.: Compressed-objective genetic algorithm. In: Runarsson, T.P., Beyer, H.-G., Burke, E., Merelo-Guervós, J.J., Whitley, L.D., Yao, X. (eds.) Parallel Problem Solving from Nature - PPSN IX. LNCS, vol. 4193, pp. 473-482. Springer, Heidelberg (2006)

6. Zhou, A., Kang, L., Chen, Y., Huang, Y.: A new definition and calculation model for evolutionary multi-objective optimization. Wuhan University of Natural Sciences 8(1B), 189-194 (2003)

7. Benedetti, A., Farina, M., Gobbi, M.: Evolutionary multiobective industrial design: The case of a racing car tire-suspension system. IEEE Transactions on evolutionary Computation 10(3), 230-244 (2006)

8. Kang, Z.: Evolution Programming and Its Application on Modeling and Forecasting, Ph. D. dissertation, Wuhan University, Wuhan, China, 36-38 (2006)

9. Rudolph, G., Agapie, A.: Convergence properties of some multi-objection evolutionary algorithms. In: CEC 2000. Proceedings of the Congress on Evolutionary Computation, pp. 1010-1016. IEEE Press, NJ, New York (2000) 
10. Guo, T., Kang, L.: A new evolutionary algorithm for function optimization. Wuhan University Journal of Natural Sciences 4(4), 409-414 (1999)

11. Zou, X.F., Liu, M.Z., Kang, L.S., He, J.: A high performance multi-objective evolutionary algorithm based on the principle of thermodynamics. In: Yao, X., Burke, E.K., Lozano, J.A., Smith, J., Merelo-Guervós, J.J., Bullinaria, J.A., Rowe, J.E., Tiňo, P., Kabán, A., Schwefel, H.-P. (eds.) Parallel Problem Solving from Nature - PPSN VIII. LNCS, vol. 3242, pp. 922-931. Springer, Heidelberg (2004)

12. Li, C.H., et al.: A fast particle swarm optimization algorithm with Cauchy mutation (to be published)

13. Laumanns, M., Thiele, L., Deb, K., Zitzler, E.: Combining convergence and diversity in evolutionary multiobjectvie optimization Evolutionary Computation, vol. 10(3), pp. 263-282 (2002) 